\title{
Single Molecule Applications of Quantum Dots
}

\author{
Thomas E. Rasmussen ${ }^{1}$, Liselotte Jauffred ${ }^{2}$, Jonathan Brewer ${ }^{1}$, Stefan $\operatorname{Vogel}^{1}$, Esben R. Torbensen ${ }^{1}$, \\ B. Christoffer Lagerholm ${ }^{1,3}$, Lene Oddershede ${ }^{2}$, Eva C. Arnspang ${ }^{1,4,5}$ \\ ${ }^{1}$ Departments of Physics, Chemistry, Pharmacy, Biochemistry and Molecular Biology, \\ University of Southern Denmark, Odense, Denmark \\ ${ }^{2}$ Niels Bohr Institute, University of Copenhagen, Copenhagen, Denmark \\ ${ }^{3}$ Weatherall Institute of Molecular Medicine, University of Oxford, Oxford, UK \\ ${ }^{4}$ National Institutes of Health, Bethesda, USA \\ ${ }^{5}$ Department of Molecular Biology and Genetics and Interdisciplinary Nanoscience Center, \\ Aarhus University, Aarhus, Denmark \\ Email: arnspang@mb.au.dk
}

Received September 25, 2013; revised October 23, 2013; accepted November 22, 2013

Copyright (C) 2013 Thomas E. Rasmussen et al. This is an open access article distributed under the Creative Commons Attribution License, which permits unrestricted use, distribution, and reproduction in any medium, provided the original work is properly cited.

\begin{abstract}
Fluorescent nanocrystals composed of semiconductor materials were first introduced for biological applications in the late 1990s. The focus of this review is to give a brief survey of biological applications of quantum dots (QDs) at the single QD sensitivity level. These are described as follows: 1) QD blinking and bleaching statistics, 2) the use of QDs in high speed single particle tracking with a special focus on how to design the biofunctional coatings of QDs which enable specific targeting to single proteins or lipids of interest, 3) a hybrid lipid-DNA analogue binding QDs which allows for tracking single lipids in lipid bilayers, 4) two-photon fluorescence correlation spectroscopy of QDs and 5) optical trapping and excitation of single QDs. In all of these applications, the focus is on the single particle sensitivity level of QDs. The high applicability of QDs in live cell imaging experiments held together with the prospects in localization microscopy and single molecule manipulation experiments gave QDs a promising future in single molecule research.
\end{abstract}

Keywords: Quantum Dots; Single Particle Tracking; Fluorescence Correlation Spectroscopy; Optical Tweezers

\section{Introduction}

Fluorescent nanocrystals composed of semiconductor materials are regularly referred to as quantum dots (QDs) because of their optical properties. Quantum confinement within the core material leads to the fluorescence emission wavelength being dependent on the core size and material composition. These days QDs are regularly being used in a variety of biological applications both in vitro and in vivo (References). Almost 15 years after their initial application in biology [1], QDs have found use in a multitude of different applications due to their superior optical properties (brightness and resistance to bleaching) as compared to conventional fluorophores. In this review we present basic concepts of QDs with a focus on the optical properties that make QDs so special and further give a survey of single QD applications in biology. QDs have a higher brightness because of their very high absorption excitation coefficients and high quantum yield. QDs also display a higher resistance to- wards photobleaching since their inorganic nature makes them less prone to degradation resulting from photoinduced bond cleavage. The resulting fluorescence lifetime (20 - $50 \mathrm{ns)} \mathrm{[2]} \mathrm{and} \mathrm{high} \mathrm{signal} \mathrm{to} \mathrm{noise} \mathrm{ratio} \mathrm{make}$ them ideal for time-resolved microscopy studies. Section 2 is an introduction to the material composition, quantum physics and optical properties of QDs. In Section 3, there are examples of two single particle applications of QDs: QDs targeted to a protein of interest followed by high speed single particle tracking (SPT) of the QD signal in live cells, and QDs targeted to a DNA-lipid analogue and SPT in lipid bilayers. In Section 4, there is an example of the use of QDs as probe in two-photon fluorescence correlation spectroscopy in which the size of the QD is found. In Section 5 we present how the use of QDs as a force-handle as well as a visualization probe using an optical tweezers assay. In all the applications mentioned in this review, the focus is on the use of QDs in single particle detection experiments. 


\section{Quantum Dots-Fluorescent Semiconductor Nanocrystals}

Quantum dots (QDs) are inorganic fluorescent nanoscale crystals with considerable enhanced optical properties in terms of brightness, photostability, blinking and bleaching compared to conventional organic and protein fluorophores. QD core sizes are in the range 1 - $10 \mathrm{~nm}$ and the individual nanocrystals contain from a few hundred to several thousands of atoms each. The core of the QD nanocrystals is typically composed of binary mixtures of semiconductor materials $(\mathrm{ZnS}, \mathrm{CdS}, \mathrm{CdSe}, \mathrm{InP}, \mathrm{CdTe}$, $\mathrm{PbS}, \mathrm{PbTe}$ ). Early QDs were composed of only a naked core but this led to inherent problems with fluorescence output due to interaction of the exciton with the surroundings. Increasing QD yield and efficiency was achieved by adding a second shell layer semiconducting material to the core resulting in a so-called core/shell QD $[3,4]$. Core-shell QDs is the main type used today in biological applications.

QDs preserve some bulk properties from the material they are made of, but because of their small size they also retain new unique physical, chemical and electronic properties due to quantum confinement effects. Probably the most amazing feature of the confinement effects is that the emission bands of QDs are dependent of the composition and size. If semiconductors are exposed to light excitation, the mobility of electrons in the material increases. Characteristic for semiconductor materials is that they have an unoccupied energy band called the conduction band and an occupied energy band called the valence band. When irradiated an electron from the valence band can get excited and promoted to the conduction band. This result in the formation of a positive charge called a hole and both the electron and the hole are free to move around the bulk material kept together by a Coulomb attraction. Together the electron and hole constitute an electron-hole pair also known as an exciton, the average distance between the electron and hole is known as the Bohr radius of the exciton. Because the excitons can be seen as particles in a box the following quantum mechanical calculations can be made. The Hamiltonian for a spherical QD of diameter $\mathrm{R}$ is given by $[5]$ :

$$
\hat{H}=-\frac{\hbar}{2 m_{e}} \nabla_{e}^{2}-\frac{\hbar}{2 m_{h}} \nabla_{h}^{2}+\frac{e^{2}}{4 \pi \varepsilon\left|r_{e}-r_{h}\right|}
$$

The first two terms are the kinetic energy operators of the electron and the hole with masses $m_{e}$ and $m_{h}$ respectively. The last term denotes the potential energy interaction of the electron and the hole that are in the positions $r_{e}$ and $r_{h}$ from the center of the sphere. Due to the charge on the electron $(-e)$ and the hole $(+e)$ the Coulombic attraction between the electron and the hole, where $\varepsilon$ is the permittivity of the material and $\left|r_{e}-r_{h}\right|$ is the distance between the hole and the electron. Solving the Schrödinger yields the following equation for the approximation of the exciton energy [5].

$$
E_{e x}=\frac{h^{2}}{8 R^{2}}\left(\frac{1}{m_{e}}+\frac{1}{m_{h}}\right)-\frac{1.8 e^{2}}{4 \pi \varepsilon R}
$$

The equation states that the exciton energy decreases with decreasing values of $\mathrm{R}$ and the term on the right will decrease with small R-values and therefore the exciton energy is largely of kinetic nature. Therefore the electrical conductivity and creation of mobile charge carriers depends on the size of the quantum dot. As a result the fluorescence emission wavelength of the QD can be tuned by size, such that the emission of smaller QDs will be blue shifted while the emission of larger QDs will be red shifted. In Figure $\mathbf{1}$ is a picture of a series of QDs emitting at wavelengths from $490 \mathrm{~nm}$ to $650 \mathrm{~nm}$.

\subsection{Optical Properties of Quantum Dots}

QDs distinguish themselves in several ways compared to conventional organic and protein fluorophore molecules. While the band gap of organic fluorescent dyes is defined by the $\pi$-electron system of the molecule as well as electron donating and withdrawing groups, the QD band gap is determined by the size of the nanocrystal even though the material remains the same. Further, many of the most commonly applied fluorophores are characterized by relative narrow emission excitation and emission spectra. QDs on the other hand have a much wider excitation range while they exhibit very narrow and symmetrical emission spectra separated by a distinct Stokes shift.

A lot of effort has been done in order to tune QD emission to cover as large a wavelength range as possible. Most commercial available QDs have emission spectra

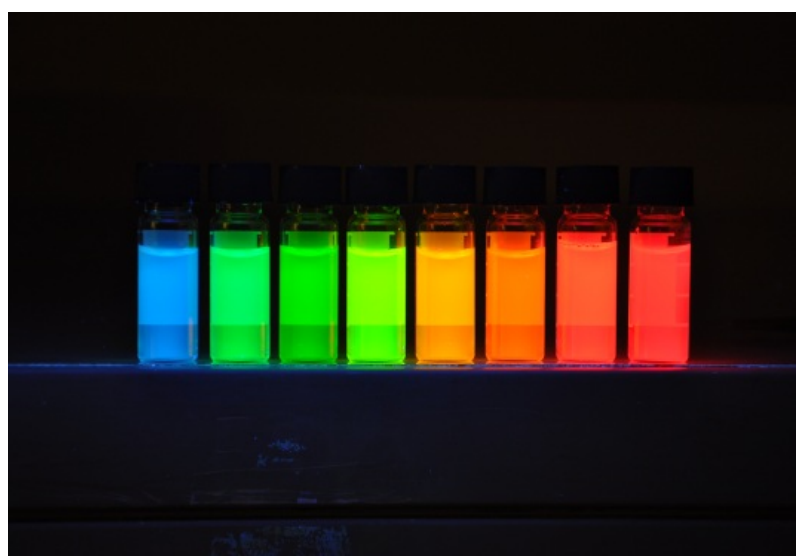

Figure 1. Image showing fluorescence emission of $\mathrm{CdSe} / \mathrm{ZnS}$ with increasing size $(490 \mathrm{NC}, 525 \mathrm{NC}, 565 \mathrm{NC}, 585 \mathrm{NC}, 605 \mathrm{NC}$, $625 \mathrm{NC} \& 650 \mathrm{NC}$ ) and cadmium-free InGaP (700NC) Quantum dots from eBioscience. 
situated in the visual part of the electromagnetic spectrum and include QD materials such as $(\mathrm{CdS}, \mathrm{CdSe}$, CdTe). QDs emitting in the Ultra Violet range have mainly been made of $\mathrm{ZnS}$ and $\mathrm{ZnSe}$ while emission in the near infrared range has been accomplished with materials such as $\mathrm{CdS} / \mathrm{HgS} / \mathrm{CdS}$, InP, InAs [6]. Mainly the latter has received much attention in the development of new QD types since the penetration of NIR light into tissues is significantly higher than shorter wavelengths of light, which are susceptible to significant scattering and absorption by the tissue. Furthermore autofluorescence from biological samples is less present in this part of the spectrum resulting in a lower fluorescent background. In Figure 2 is an overview of emission wavelengths of commercially available QDs.

QDs display one more characteristic optical feature. QDs blink, periodically switching between an on and off luminescent state. The cause of this behaviour is due to trapping and untrapping of charges due to surface defects, this enables the distinction of single QDs, which is essential to perform single molecule measurements.

\subsection{Blinking of Quantum Dots}

A major disadvantage of QDs is that the fluorescence emission is intermittent, i.e., the QDs fluctuate between a fluorescent emitting bright state and a non-emitting dark, with the dark non-fluorescence emitting states lasting for periods in the ms to second range timescale. For example, commercially available hybrid CdSe/CdTe QDs have been shown to spend a majority of their time in a nonfluorescent dark state [7-13]. The current consensus for this observed QD intermittency is that non-charged QDs are fluorescent, while charged QDs are not [8,14]. The observed intermittency has further been shown to be partially inhibited by small reducing agents such as $\beta$-mercaptoethanol (BME), dithiothreitol (DTT) and mercaptoethylamine (MEA) in $\mathrm{mM}$ concentrations $[9,15,16]$. Decreased blinking has also been accomplished by growth of thick semiconductor shells around the QD cores [17,18]. Non-blinking $\mathrm{CdZnSe/ZnSe} \mathrm{QDs} \mathrm{have}$ also been reported, however, these QDs have a very broad emission spectra with three characteristic emission peaks making them non-useable for multiplexing applications [14].

When this fluorescence blinking of QDs was first observed, it was a surprise, since there was no known quantum physical mechanism which could explain this [19]. What was furthermore a surprise was that the off times of QDs were distributed according to an inverse power law rather than an exponential decay [20]. An exponential dependence of the probability $\mathrm{P}$ that a $\mathrm{QD}$ is off for the time $\tau$ would follow the equation $\left(=\left(P \propto e^{-\tau \alpha}\right)\right.$, where $\tau$ is the off time and $\alpha$ the slope of the linear plot on a semilog scale. A power law dependence of the probability $\mathrm{P}$ that a QD is off for the time $\tau$ would follow the equation $\left(P \propto \tau^{-\alpha}\right)$, where $\alpha$ is the slope of

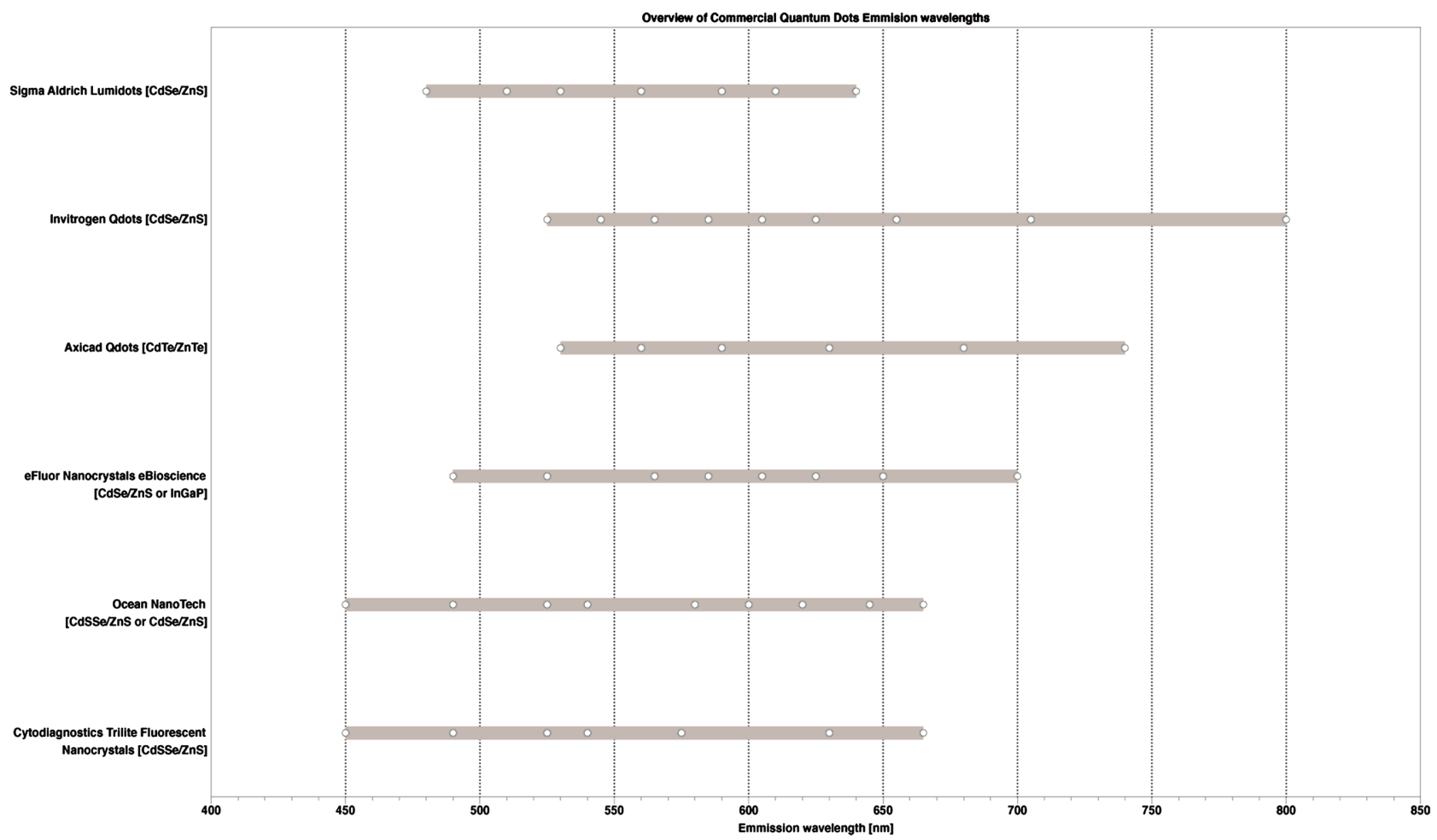

Figure 2. An overview of emission wavelengths of quantum dots from different commercial sources. 
the linear plot on a log-log scale. The power law dependence of the blinking of QDs is unchanged for temperatures from $10 \mathrm{~K}$ to room temperature, for core radii from $15 \AA$ to $27 \AA$, for different materials: CdSe, TeSe, InP, for laser intensities from $100 \mathrm{~W} / \mathrm{cm}^{2}$ to $20 \mathrm{~kW} / \mathrm{cm}^{2}$ and pressure from atmospheric pressure to vacuum [21-24]. Because QDs consist of two alloy compositions and the bond length in the crystal lattice of the one alloy is different from the bond length in the crystal lattice of the other alloy there are imperfections in the crystal structure. The quantum physical explanation of the blinking of the QDs proposes that the intermittency is caused by ionization of the QD. After photon excitation, two electron-hole pairs could be excited simultaneously. One pair could recombine by the Auger effect and emit one hole or one electron outside the QD. This is also called a trapped state as the emitted electron will be trapped in the surrounding medium for some time. During this off-time the left behind electron hole in the QD will generate a very fast non-radiative Auger channel for any new excited electron-hole. The electron hole will remain inside the QD during the off-period due to different barrier heights for electron and hole. The result is that the radiative luminescence is quenched during the off period, corresponding to the dark state of QDs $[25,26]$.

\subsection{Bleaching of Quantum Dots}

A major attraction of QDs is that they are more resistant to photobleaching than is conventional fluorescent dye and protein fluorophores. But QDs have also been shown to photobleach under intense laser illumination [27,28] and more recently even with $\mathrm{Hg}$ arc lamp illumination [29]. However, contrary to fluorescent dyes and proteins that display single step photobleaching from a fluorescent emitting state to a dark state, QDs have been shown to photobleach by a gradual blue-shifting of their emission color eventually reaching a permanent dark state $[8,27,28]$. This photobleaching of QDs is indicative of a process in which the QD core is gradually shrinking as a result of photooxidation of the core [8,27,28,30,31], a hypothesis which is supported by the reported slowing of blue shifting in a nitrogen atmosphere [28].

Photooxidation at the surface of QDs has shown to result in quenching of fluorescence emitted by CdSe QDs. Formation of surface quenching states will cause a decrease in emitted fluorescence. The bleaching of QDs is accompanied with a blueshift in the emission spectrum. This bleaching process has been shown with laser powers of $20 \mathrm{~kW} / \mathrm{cm}^{2}$ and in both air and nitrogen atmospheres. In nitrogen atmosphere the bleaching is slower but still occurs [28]. It is possible to prevent the bleaching and blueshifting of QDs by adding $\beta$-mercaptoethanol (BME)
[32] or mercaptoethylamine (MAE) [29]. Figure 3 depicts images of QD655s that were illuminated under aqueous conditions for a few minutes with and without BME added. Left and rightwimages were acquired about 7 minutes apart after continuous illumination with blue filtered light. In the absence of BME, the QD655 emission color was observed to shift from an initial orange/red hue to a yellow-green hue. In the presence of 25 $\mu \mathrm{M}$ BME, the QD655 emission color was observed to be significantly stabilized to an orange/red hue for the duration of the experiment [32].

Bleaching of QDs happens at a slower rate than other standard fluorophores. A direct comparison has been made between Alexa488 and QDs. Alexa488 fades after 60 seconds of constant illumination whereas the QDs are still emitting after 180 seconds. There are also many examples of longer full intensity periods of QDs [33].

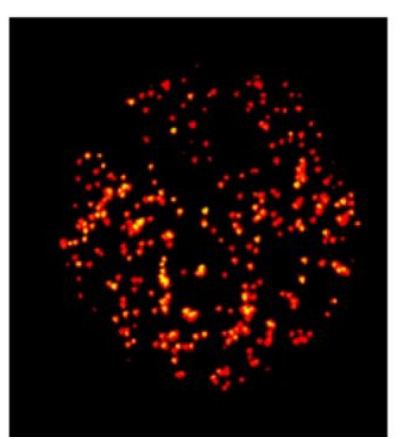

(a)

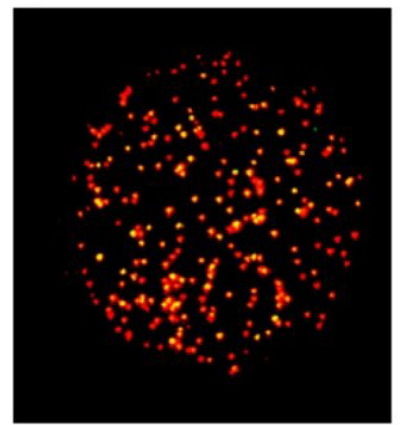

(c)

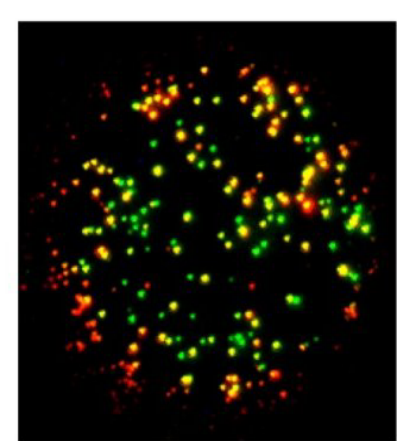

(b)

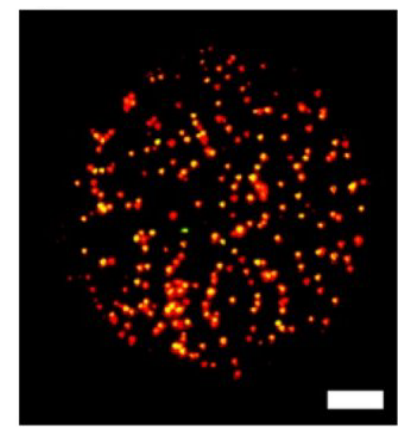

(d)
Figure 3. Fluorescence color switching of QDs emitting at $655 \mathrm{~nm}$. QDs were non-specifically adsorbed to a glass coverslip and imaged under aqueous conditions on a CoolSNAP-Pro $_{\text {cf }}$ color CD camera with 10 sec integration time. Left and right images were acquired about 7 minutes apart after continuous illumination with blue filtered light from a $100 \mathrm{~W} \mathrm{Hg}$ arc lamp. (a, b) QD655 in the absence of $\beta$-mercaptoethanol (BME), the QD emission color was observed to shift from an initial orange/red hue to a yellow-green hue. (c, d) QD655 in the presence of $25 \mu \mathrm{M}$ BME, the QD emission color was observed to be significantly stabilized to an orange/red hue for the duration of the experiment (scale bar is $1 \mu \mathrm{m}$ ) Reprinted with permission from [32]. 


\subsection{Quantum Dot Coatings and Bioconjugation}

In order for QDs to be used in single molecule applications certain surface modifications have to be done to the bare core/shell QDs. Both core-shell and core only QDs are coated with a thin layer of an organic ligand such as trioctylphosphine oxide (TOPO) as a result of the organic synthesis route that is used to make them [34,35]. While a hydrophobic ligand such as TOPO stabilizes the QDs in an organic solvent, these QDs need to be made more water soluble for biological relevant applications [34-38]. Keeping the original ligands in place gives the brightest QDs, and one way to do this is to use an amphiphilic co-block polymer (e.g. an octylamine-modified polyacrylic acid) to coat the QDs [38]. The hydrophobic side chains of these polymers interdigitate with the organic ligands on the QDs, and the hydrophilic part constitute the new surface of the now water soluble QDs, adding another $1-2 \mathrm{~nm}$ to the diameter [39,40]. The hydrophilic ends of these QDs have reactive groups that are available for further bio-conjugation needed in order to direct the QD binding towards a specific target. Bio-conjugation of the QD will add to its final size and typical fully biofunctional QDs have a size of $\sim 20 \mathrm{~nm}$.

Water stabilized QDs preferentially have reactive chemical groups on the surface that are available for biofunctionalization necessary in order to direct the QDs towards a specific target for biological applications [36]. Often, the initial chemical groups on the surface are carboxylic groups, but these can be reacted with e.g. diamine polyethylene glycols (PEG), resulting in amino functionalized QDs. Covalent bio-functionalization of carboxylic or amine QDs is easily achieved by chemical cross-linkers, and various strategies exist depending on the conjugation partners [40,41]. For example, the cross-linker EDC (1-ethyl-3-(3-dimethylaminopropyl)carbodiimide) can be used to conjugate carboxylic groups on QDs to amine groups on e.g. proteins or peptides [42,43]. Another bifunctional cross-linker SMCC (succinimidyl-4( $N$-maleimidomethyl)cyclohexane-1-carboxylate), which has a maleimide reactive group and an NHS ester, can be used to couple thiols on e.g. (mildly and selectively) reduced antibodies or antibody fragments to amines on QDs [44]. QD conjugation of two amine groups has also been done using Traut's reagent and $N$-succinimidyl iodoacetate, a hetero bifunctional cross linker which couples thiols and amines (SIA) [40]. Often, a linker such as PEG is introduced between the QD and the bio-molecule to increase steric freedom, and to minimize unspecific binding of the QDs $[45,46]$. Figure 4 illustrates several conjugation strategies between QDs and organic molecules.

\section{Single QD Applications in Biology}

The enhanced optical properties of QDs, in particular the significant brightness and photostability, make these materials highly suitable for use in biological applica- tions requiring even single molecule sensitivity.

\subsection{Single Particle Tracking (SPT)}

The foremost single molecule application where QDs have been used is single particle tracking (SPT) [48]. In SPT, single molecules of interests (MOIs) are sparsely labeled with a luminescent or scattering probe and the movement of the MOI:probe complexes is imaged by time-lapse microscopy at repetition rates ranging from a few to $50,000 \mathrm{~Hz}$ depending on the process investigated and the signal of the probe [48]. In this way, trajectories describing the motion of single MOI:probe conjugates can be constructed with nanometer precision and millisecond time resolution to provide details of the molecular dynamic that ensures cellular structure and function. More detail on technical aspects and data analysis in SPT can be found in recent reviews (e.g. [48])

The most common MOIs in SPT for biological applications are amphiphilic molecules such as lipids, lipid anchored proteins, and transmembrane proteins. In this case, the investigated motion of the MOIs is most frequently restricted to lateral diffusion in two dimensions within the plane of e.g. the plasma membrane or in a model membrane. In this particular application more leeway in the choice of probe is given because the limiting factor in the lateral motion of the MOI:probe complex is the viscosity of the membrane within which the hydrophobic part of the MOI is residing. This is because the viscosity of the membrane is 100 times that of viscosity in the surrounding aqueous solution. For this reason it is generally accepted that even probes that are much larger than a particular MOI will only have a minor impact of the motion of the MOI. In contrast, in the case of an aqueous soluble MOI the motion will be severely affected by large probes. In fact even fluorescent proteins, e.g. green fluorescent protein which has a molecular weight of about $28 \mathrm{kDa}$ and a hydrodynamic radii of about $3.4 \mathrm{~nm}$ will have a dramatic effect on the motion of most biological MOIs.

Initially SPT experiments were done using interfereence contrast video microscopy measuring the scatter from micrometer-sized latex beads or $40-100 \mathrm{~nm}$ gold nanoparticles [49]. Subsequently the technique has been extended to track single fluorescent dyes and proteins (sometimes called single molecule fluorescent tracking, SMFT) [50]. By studying the motion of single molecules, different modes of motion can be distinguished, and often the motion turns out to be very heterogeneous in a way that cannot be described by ensemble measurements which involve averaging over a large pool of indistinguishable molecules [51]. However, neither the latex 


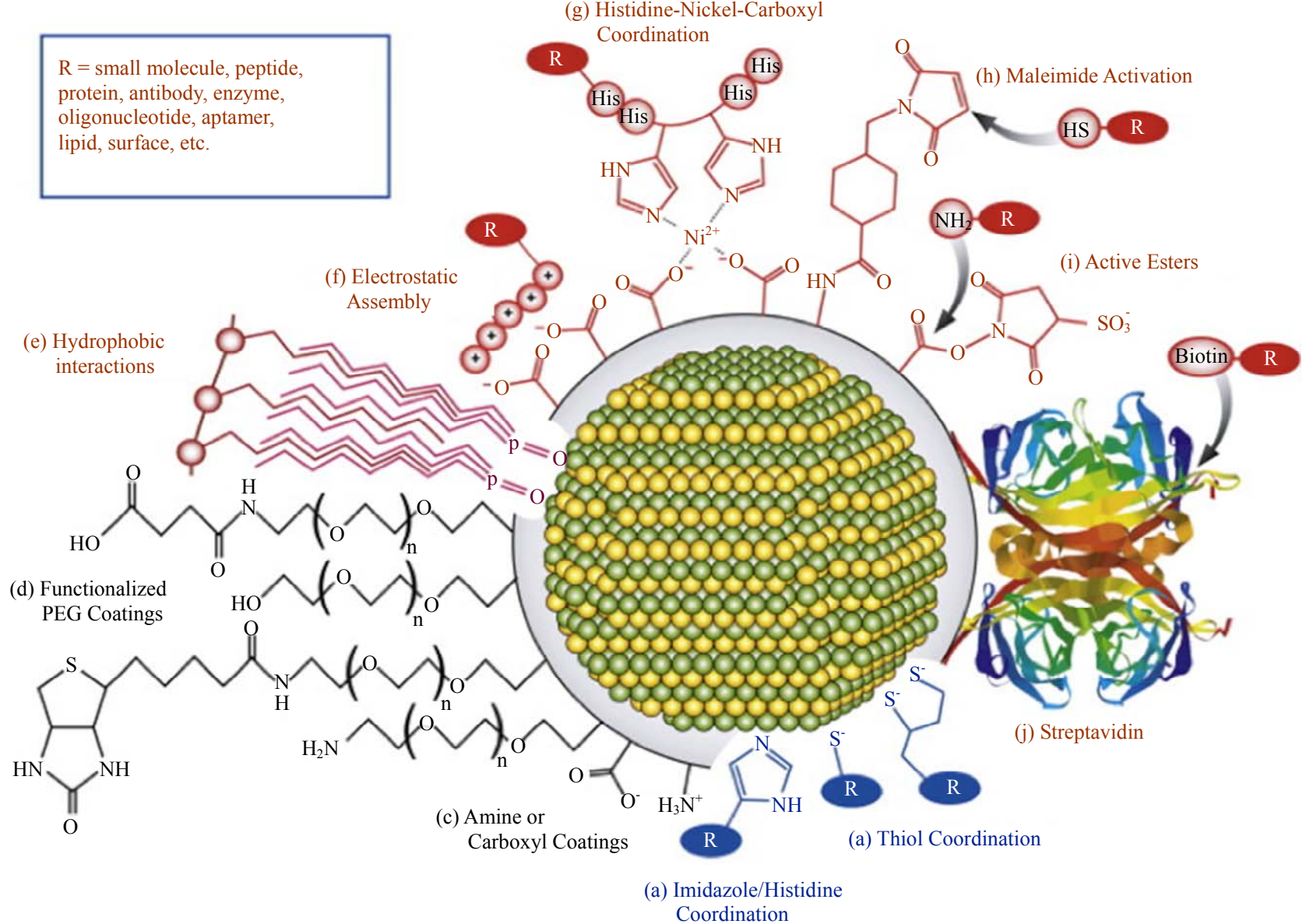

Figure 4. An illustration of some selected surface chemistries and conjugation strategies that are applied to QDs. The grey periphery around the QD represents a general coating. This coating can be associated with the surface of the QD via (e) hydrophobic interactions, or ligand coordination. Examples of the latter include: (a) monodentate or bidentatethiols, (b) imidazole, polyimidazole (e.g. polyhistidine), or dithiocarbamate (not shown) groups. The exterior of the coating mediates aqueous solubility by the display of (c) amine or carboxyl groups, or (d) functionalized PEG. Common strategies for bioconjugation include: (a) thiol modifications or (b) polyhistidine or metallothionein (not shown) tags that penetrate the coating and interact with the surface of the QD; (f) electrostatic association with the coating; (g) nickel mediated assembly of polyhistidine to carboxyl coatings; (h) maleimide activation and coupling; (i) active ester formation and coupling; (j) biotin-labeling and streptavidin-QD conjugates (not to scale) Reprinted with permission from [47].

beads or gold particles nor the fluorescent dyes or proteins are ideal probes for SPT [52]. The former suffer from being bulky, and the latter have limiting optical properties, and none of them are ideal for multiplexing studies. QDs on the other hand, are a great compromise between those two categories of probes. They have a moderate size, an extreme brightness, and an excellent resistance to photobleaching, and are ideal for multiplexing studies even with simple setups [53]. The ease, by which they are bio-functionalized, further makes it possible to direct their binding towards almost any molecular target of interest, and makes them a preferred choice for SPT (or single quantum dot tracking, SQT) studies [40].

In a typical SPT experiment, the motion of sparsely labeled single molecules is monitored by recording a time-lapse image series [54,55]. Subsequently, the individual main intensity peaks of the diffraction limited point spread functions from the well separated probes are detected and fitted computationally to a 2D Gaussian distribution in order to localize the centroid positions with a sub-diffraction limited spatial resolution of $10-40$ $\mathrm{nm}$. These centroid positions are then linked between successive frames using advances linking algorithms to build up single molecule trajectories [56-58]. In the case of tracking QDs these algorithms take QD blinking into account and are able to track molecules even if the QD is "off" for some frames. The resulting trajectories are typically analyzed by calculating the mean squared displacement (MSD), or alternatively by calculating the probability distributions of the squared displacements [54,55]. For this analysis, QDs has the advantage that the 
trajectories are long enough to allow for analysis of the single trajectories. This is in contrast to tracking with fluorescent dyes or proteins where the limited photostability of these probes requires that all trajectories are pooled to give an analysis averaged in space and time hindering the observation of transient and rare events. By the shape of a MSD-vs-time plot it is possible to classify the mode of motion. A freely moving molecule undergoing Brownian motion will be a straight line in an MSDvs-time plot, according to MSD $=4 \mathrm{D}$, with $\mathrm{D}$ being the diffusion coefficient. Normally, however, plasma membrane molecules in live cells experience hindrance in their motion. This results in a MSD curve with a steep slope at small times and a more moderately increasing slope or a flat slope at longer times, indicating a time dependent diffusion. The reasons for the confinement are many, including interactions with membrane domains, cytoskeleton barriers, molecular crowding, membrane topology, and specific interactions with other membrane molecules [59-62].

Since the first paper on tracking single QDs appeared 10 years ago [63], many have followed, and contributed to the present understanding of structural, dynamical, and functional aspects of the plasma membrane. Studies have shown heterogeneous motion of individually labeled plasma membrane proteins and lipids, and that e.g. actin [64], the extracellular matrix [65], lipid microdomains $[66,67]$, and cholesterol all affect the movement of these molecules. In neurobiology, single QD studies have been used extensively to study the motion of specific receptors in synapses under various cellular conditions and stimuli (for review see [68]). For instance, it was shown how $\mathrm{GABA}_{\mathrm{A}}$ receptors distribute asymmetrically across the axon growth cone in a microtubule and calcium dependent manner in response to a GABA gradient [69], and how AMPA receptor mobility is functional in recovery of synaptic activity [70]. Details of non-neural signal transduction pathways have also been revealed. Lidke and co-workers tracked EGF-conjugated QDs targeting the EGF receptor (erbB1) that is often found dysregulated in many cancers. Upon QD-ligand binding homo/ hetero dimerization and endosomal uptake was followed, and further, a previously unknown mechanism of retrograde transport of the QD-EGF-EGFR complex from the filopodia to the cell body was found [71,72]. Tracking of membrane species using orthogonal multicolor QD labeling strategies have been conducted tracking the same membrane species [64,73-76]. Recently, Clausen et al. has extended this to the orthogonal and simultaneous tracking of three different species, a lipid, a lipid-anchored protein, and a transmembrane protein (In press PLOS ONE). Most QD tracking experiments are recorded at video rate $(25$ or $30 \mathrm{~Hz})$, however, using a camera with fast read-out, the extreme brightness of QDs allows for imaging at up to $1750 \mathrm{~Hz}$ [77].

\subsection{Single Particle Tracking of Hybrid Lipid-DNA Analogues Using Quantum Dots}

Labelling specificity is a major concern when performing SPT experiments. Preferably the conjugation system used to couple QDs to the target biomolecule should exhibit high specificity and strong avidity towards the target and as high degree of monovalency as possible. Another consideration when designing a conjugation system for SPT could be to make it as interchangeable as possible so the same system could be designed to bind different sized QDs to different targets enabling easy labelling for multicolor SPT experiments. As a result Vogel et al. have synthesized lipid-DNA analogues based on a polyaza crown ether depicted in Figure 5 [78]. The membrane anchors are linked to the nitrogens of the polyaza crown ether building block and can be interchanged to encompass either acyl chains or sterols such as cholesterol, which in turn can be used to probe different environments in the plasma membrane or an artificial membrane system. The lipid-DNA analogue is then coupled to an oligomer of 17 bases at the 3 ' end, which in turn can bind to a complimentary strand that has a 5'-biotinylated oligomer. Finally, streptavidin coated QDs can be bound to the biotin end of the complimentary strand with high specificity and avidity forming the SPT complex. The inherent advantage of this conjugation system is the specificity of the DNA complexation, since several different lipid-DNA analogues with different membraneanchor moieties could be labeled individually by using unique DNA sequences that bind only to certain colored QDs.

\subsection{Single Particle Tracking Studies in Supported Lipid Bilayers}

SPT studies were conducted to investigate the application of previously mentioned lipid-DNA analogues in supported lipid bilayers made from a binary lipid mixture of the phospholipids 1,2-Dipalmitoyl-sn-Glycero-3-phosphocholine (DPPC) and 1,2-Dioleoyl-sn-Glycero-3-phosphocholine (DOPC) in the ratio 1:1 supported on glass coverslips. The supported lipid bilayers were made as described in [79], briefly $24 \mathrm{~mm}$ in diameter glass coverslips were cleaned in a basic piranha solution composed of Milli-Q water, ammonia and hydrogen peroxide in the ratio $6.2: 1: 1$ respectively and heated to $100^{\circ} \mathrm{C}$ for two hours. The processed cover slips were rinsed 3 times in Milli-Q water and dried in the oven prior to use. The phospholipids DPPC and DOPC (Avanti Polar Lipids) were dissolved in a mixture of $90 \%$ chloroform and $10 \%$ acetonitrile at a total lipid concentration of $10 \mathrm{mM}$. In order to visualize the membranes in the microscope 0.5 

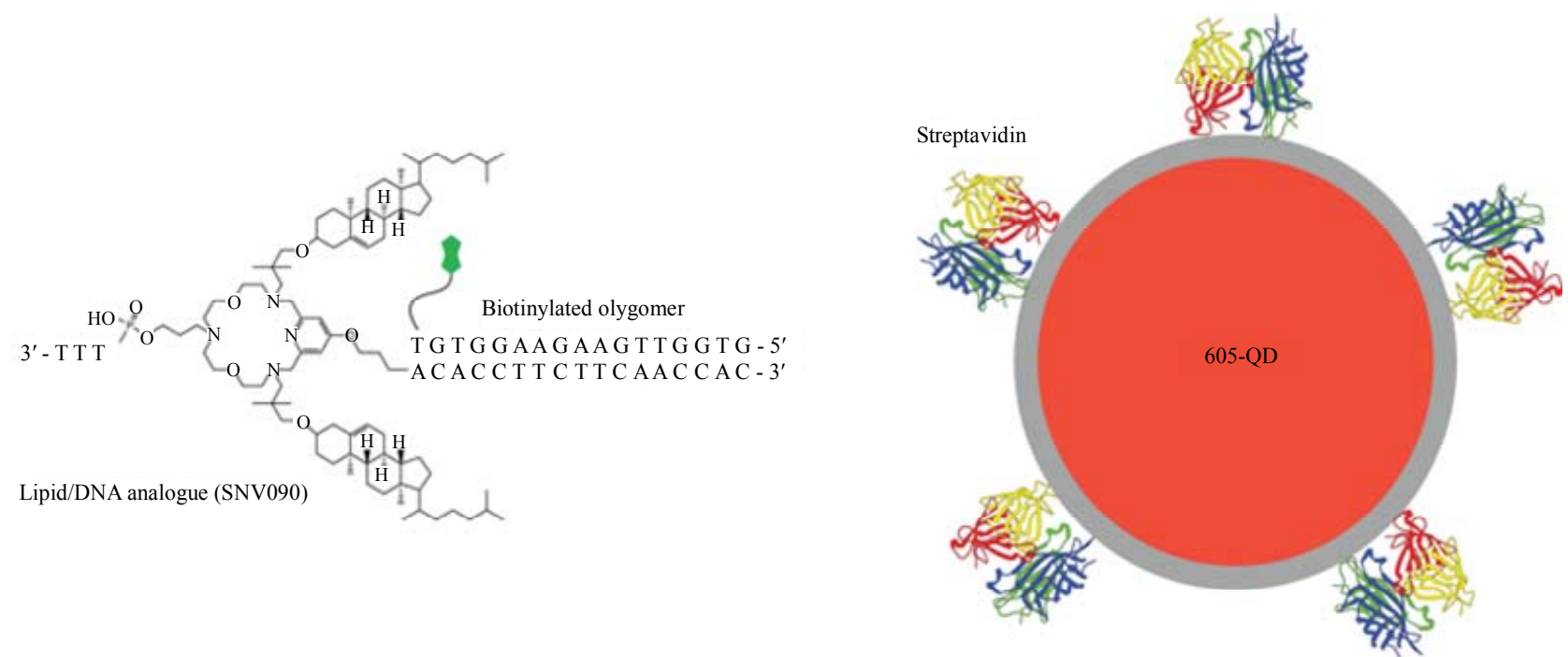

Figure 5. Schematic representation of the Streptavidin-coated 605 QD coupled to the lipid-DNA analogue by a biotinylated complementary oligomer (not drawn to scale).

mol\% of the fluorescent lipid dye NBD-PC (Avanti Polar Lipids) was added to the lipid mixture. The supported lipid bilayers were prepared on the glass cover slips by spincoating using a Chemat technology spincoater KW4A. Spincoating is performed by adding $50 \mu \mathrm{l}$ of the lipid solution is placed in the middle of the coverslip and was done using the following settings:

\begin{tabular}{ccc}
\hline \multicolumn{3}{c}{ Spincoater settings } \\
\hline Steps & Duration & Speed \\
1 & $3 \mathrm{sec}$. & $500 \mathrm{rpm}$ \\
2 & $40 \mathrm{sec}$. & $3000 \mathrm{rpm}$ \\
\hline
\end{tabular}

Afterwards the spincoated coverslips are stored in a vacuum desiccator for at least 24 hours to ensure the solvent has evaporated. Depending on the lipid concentration and of the applied volume of stock solution it is possible to form several bilayer on top of each other. Hydration of the dry spincoated bilayers is accomplished by placing the spin-coated coverslip into a special heating stage composed of an aluminum holder and is fixed in place by a plastic ring. The hydration of the multilaminar bilayer system enables one to wash of the top layers by gentle pipetting of the revealing the bottom bilayers on the coverslip. Heating of the setup is essential since the phase transition of the lipid mixture is above $\mathrm{RT}$ and it also aides in removing the top bilayers due to the increased mobility of the lipids in the bilayers. The hydration of the DOPC:DPPC 1:1 supported lipid bilayers was performed in Dulbecco's phosphate buffered saline (PBS) (Sigma Aldrich) and the sample was heated to $60^{\circ} \mathrm{C}$ well above the phase transition of the mixture and left to incubate for 30 minutes to ensure the mem- brane is its fluid state. Gentle pipetting was used to remove excess lipid and to reveal the bottom bilayers on the coverslip. Below the phase transition of the lipid mixture DOPC should phase segregate to form ridged flower-shaped domains in which the fluorescent dye NBD-PC will not be present. Our microscopy studies have shown that these flower-shaped domains do not occur in the bottom lipid bilayer, possibly due to interacttions between the glass and the lipids as well as due to the roughness of the glass surface. Therefore SPT studies were only performed in bilayers that exhibited the flower-like domains upon cooling below $40^{\circ} \mathrm{C}-36^{\circ} \mathrm{C}$.

Once a planar patch of membrane has been established and characterized by fluorescent microscopy $100 \mu \mathrm{l}$ of a $100 \mathrm{pM}$ lipid-DNA analogue was added locally and incubated for 30 minutes. The PBS was exchanged in order to remove any non-incorporated membrane anchors. Afterwards $100 \mu \mathrm{l}$ of a $100 \mathrm{pM}$ biotin coupled complimentary strand DNA solution was added and left to incubate for 15 minutes. The PBS was exchanged in order to remove any unbound excess complimentary DNA, followed by the addition of $10 \mu \mathrm{l}$ of a $1 \mathrm{nM}$ streptavidin coated QD605 solution (Invitrogen), which was left to incubate for $10 \mathrm{~min}$. The unbound QDs were removed by a final washing step.

SPT studies were done by first bleaching away the signal from the NBD-PC in order to visualize the QDs bound to the membrane surface, see Figure 6. Thereafter, varying lengths ( $300 \& 900$ frames) were recorded and from these the individual trajectories of the QDs were extracted using ImageJ and a plugin called Spot tracker. The mean squared displacements of these trajectories were calculated using Wolfram Mathematica.

From the preliminary results obtained from the SPT 

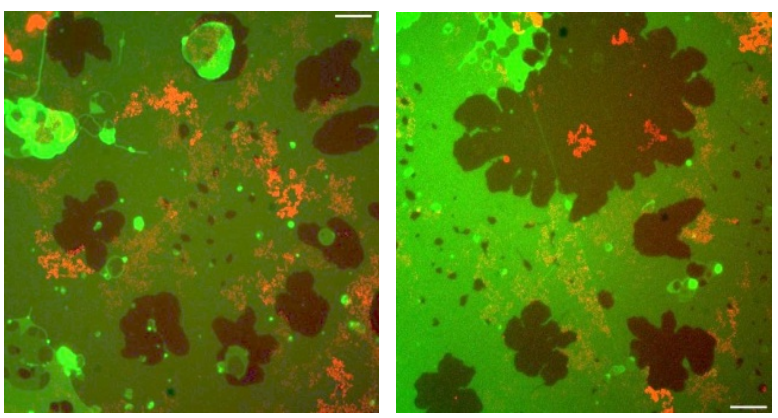

Figure 6. Epi-fluorescence images showing the membrane (shown in green) labelled with $1 \mathrm{~mol} \%$ NBD-PC and superimposed tracks of QD605 coupled to the lipid-DNA analogue imbedded in the membrane (shown in red) (scalebar is $8 \mu \mathrm{m}$ ). The diffusion coefficient outside domains was 1.84 $\pm 1.08 \mathrm{\mu m}^{2} / \mathrm{sec}$ whereas the diffusion coefficient of molecules in contact with domains was $1.22 \pm 0.00 \mu \mathrm{m}^{2} / \mathrm{sec}$. The diffusion coefficient inside domains was $0.42 \pm 0.20 \boldsymbol{\mu m}^{2} / \mathrm{sec}$.

experiments three distinct diffusion patterns have been observed: unhindered diffusion outside domains, hindered diffusion at the border of the liquid and gel phase domains and finally unhindered diffusion inside the gel domains. It was unexpected to observe diffusion in the supposedly immobile DPPC gel phase present in the flower shaped domains - most likely this is due to the phase not being completely equilibrated. As to be expected, the calculated 2D diffusion coefficients decrease in relation to the amount of contact the SPT complex has to the immobile phase. The most predominant diffusion events were observed to be the unhindered diffusion in the liquid phase. There were several observations of diffusion along the edge of the flower domains. Finally, some occasional events showed the SPT complex leaving the rim of a domain at continuing the diffusion into the fluid phase, whereas diffusion from inside a domain into the fluid phase was not observed.

For biological membranes it has been reported that a typical lateral diffusion values range between $1-4 \mu \mathrm{m}^{2}$ $\mathrm{sec}^{-1}$ and relating the values from this preliminary SPT study the values seem comparable. As a control Fluorescence Recovery after Photobleaching (FRAP) was performed on the NBD-PC labeled fluid phase of the supported bilayers. The measurements were performed on both membrane systems with a confocal laser-scanning microscope (CLSM) of the type (Zeiss LSM 510). Usually when doing FRAP only a spot is bleached in order to determine the diffusion. However based on the work of Braeckmans et al. line FRAP is now possible [80]. A region of interest (ROI) was chosen to be $100 * 1$ pixels, which would be used for all measurements. Compared to ordinary FRAP this improved technique is faster due to the scanning motion and is able to determine the diffusion constant and mobile fraction in more localized areas [80].

$$
\begin{aligned}
& \frac{F(0, t)}{F_{0}}=\sum_{n=0}^{\infty} \frac{\left(K_{0}\right)^{n}}{n !} r_{0 e}\left(n r_{0 c}^{2}+\left(a_{n}-n\right) r_{0 e}^{2}\right)^{-1 / 2} \\
& F(y, t)=F(y, 0)+k(F(y, t)-F(y, 0))
\end{aligned}
$$

From the FRAP measurements the lateral diffusion coefficient of the fluid phase was determined to be $2.9 \pm$ $1.8 \mu \mathrm{m}^{2} \cdot \mathrm{sec}^{-1}$, which is significantly higher than the 1.8 $\pm 1.1 \mu \mathrm{m}^{2} \cdot \mathrm{sec}^{-1}$ calculated from SPT measurement. This is not surprising since the acquisition rate was slower in the FRAP studies. When also keeping in mind that only the mobile fraction is included in the FRAP studies, no direct comparison between these numbers. Furthermore, it cannot be excluded that the QDs studied by SPT are bound to the membrane by more than only one lipid DNA analogue. The quantum dots used for these experiments from Invitrogen have approximately 37 streptavidin binding sites on one quantum dot. Therefore cross-linking of one quantum dot to several membrane anchors can be a possibility. Although only preliminary SPT data has been collected, the application of the lipid-DNA analogue seems to be promising. Additional data needs to be collected in order to evaluate the diffusion coefficient values reported so far as well as if the lipid-DNA analogue affects the physiochemical properties of the supported bilayer. One of the most interesting applications of the anchor molecules is multi-color imaging, which can be performed with different sizes (and hence colors) of quantum dots. If the anchors could be designed to have a particular preference for a particular lipid environment such as lipid domains these could then be specifically labeled. If such a labeling probe could be developed in vitro it would be possible to label domains in cells and thus one would be able study the dynamics of lipid rafts below the diffraction limit.

\section{Quantum Dots as Probes for Two-Photon Fluorescence Correlation Spectroscopy}

Fluorescence correlation spectroscopy [FCS] is a very useful technique to study the movement and interactions of fluorescently labelled biomolecules and fluorophores. Elson, Magde et al. [81] introduced FCS in 1972 as an analogous technique to Dynamic Light Scattering and Relaxation Kinetics, in an attempt to overcome inherent limitations in both techniques [82]. In comparison with optical scattering, fluorescence is much more sensitive and selective enabling measurements with a low background intensity and detection sensitivity that spans from nanomolar concentrations to the single molecule regime. FCS can be performed on either a confocal microscope or a two-photon fluorescence microscope [83], nevertheless in both experimental setups a laser is focused down to a small focal volume in the order of a few femtoliters. 
In the confocal setup this is achieved by the confocal pinhole whereas the focal volume is inherent with a twophoton setup since only a small volume has high enough photon densities for two-photon excitation process to occur [84]. In Figure 7 is a schematic representation of a two-photon fluorescence correlation spectroscopy microscope setup.

The focal volume for a two-photon setup is defined as

$$
V_{e f f}=\left(\frac{\pi}{2}\right)^{\frac{3}{2}} r_{0}^{2} z_{0}
$$

where $r_{0}$ is the beam waist in the radial direction and $z_{0}$ is the beam waist in the axial direction. In order to determine the size of the focal volume and the values of $r_{0}$ and $z_{0}$ the setup is calibrated by measuring the diffusion of a well-known fluorescent size standard such as a fluorophore of a fluorescent polymeric bead. When fluorescent or fluorescently labelled species diffuse randomly through the focal volume due to Brownian motion the resulting fluctuation fluorescence signal is recorded as a function of time. These fluctuations can be analyzed by the use of autocorrelation function $\mathrm{G}(\tau)$ from which a diffusion value can be attained.

$$
G(\tau)=\frac{\langle\partial I(t) \partial I(t+\tau)\rangle}{\langle I(t)\rangle^{2}}
$$

where $\delta I(t)=I(t)-\langle I(t)\rangle$ is the deviation from the mean fluorescence intensity, $\langle I(t)\rangle$. The auto-correlation function for translational diffusion using two-photon excitation can be calculated as [85].

$$
G(\tau)=G_{0}(\tau)\left[1+\frac{8 \tau_{D}}{r_{0}^{2}}\right]^{-1}\left[1+\frac{8 \tau_{D}}{z_{0}^{2}}\right]^{-1 / 2}
$$

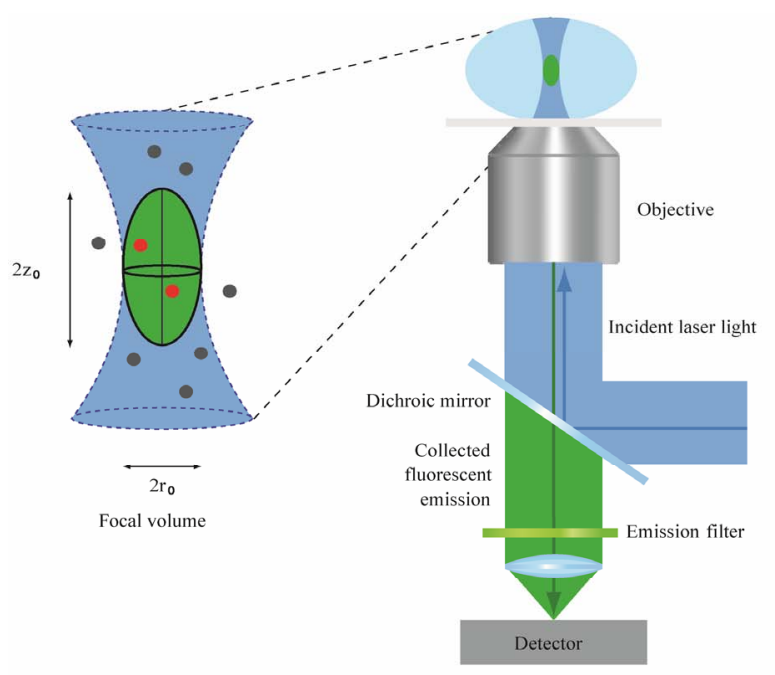

The amplitude of the autocorrelation function is denoted $G_{0}(\tau)$ and is inversely proportional to the average number of fluorescent species present in the focal volume, and therefore can be used to determine the concentration of the fluorescent species. Consequently, the amplitude will increase with low concentrations and decrease with high concentrations of the fluorescent species respectively.

$$
G_{0}(\tau)=[N]^{-1}=\left[\left\langle C_{N} V_{e f f}\right\rangle\right]^{-1}
$$

The decay rate of the autocorrelation function yields information about the occupancy of the fluorescent species in the focal volume. The function shows the probability of the fluorescent species being in the focal volume at $t=0$ and still remaining in the focal volume at a later time point $t+\tau$. Since large molecules diffuse slower than smaller molecules the probability of finding the same molecule in the focal volume at higher values of $t+\tau$ will also increase-in turn autocorrelation curves of large and small molecules will be shifted to the right and left respectively.

The diffusion coefficient can be determined by relating the diffusion time $\tau_{D}$, which is the $\tau$ value corresponding to the half value of the autocorrelation amplitude, to the following relation [86].

$$
\tau_{D}=\frac{r_{0}^{2}}{8 D}
$$

Furthermore, assuming that the diffusion molecules are spherical, the Stokes Einstein equation can be used to determine the hydrodynamic radius, $R$, from the determined diffusion coefficient, $D$

$$
D=\frac{k_{B} T}{6 \pi \eta R}
$$
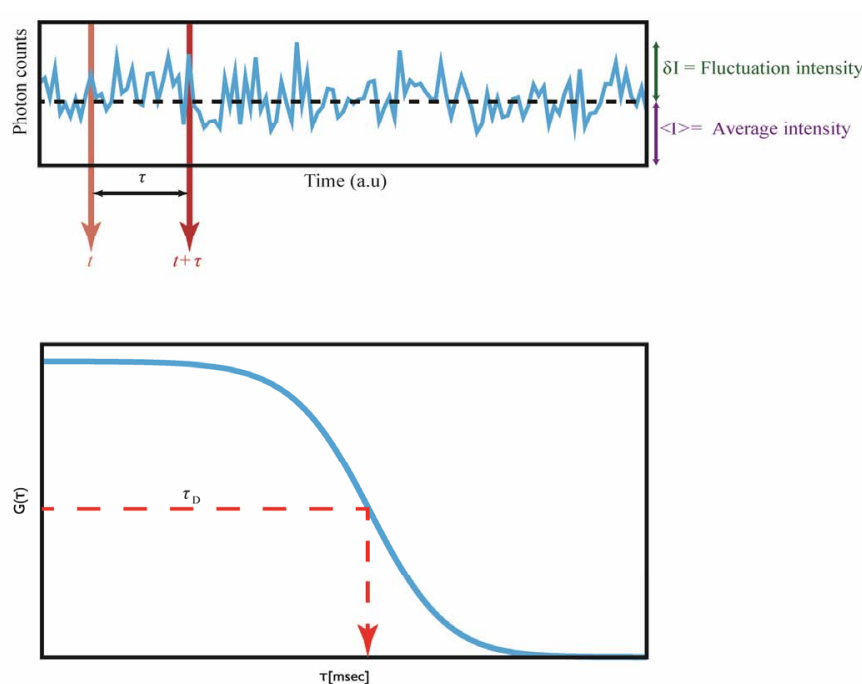

Figure 7. A schematic representation of a two-photon fluorescence correlation setup (a). Fluctuation measurements and the resulting autocorrelation analysis (b). 
where $k_{B}$ is the Boltzmann constant, $T$ is the absolute temperature (kelvin), $\eta$ is the viscosity of the medium and $R$ is the hydrodynamic radius of the diffusion molecule [87].

In Figure 8 we present auto correlation curves and fits of $605 \mathrm{sAv}$ quantum dots from Invitrogen. The FCS measurements were performed in $50 \mathrm{mM}$ sodium borate pH 8.2 with $1 \%$ (w:v) BSA at RT (293 K). The experiments were performed on a custom built system which is described in [88] and the data was fitted using the Globalssoftware package developed at the Laboratory for Fluorescence Dynamics at the University of California Irvine. The excitation wavelength was $820 \mathrm{~nm}$. It can be seen from the figure that fitting the data to the simple diffusion model (equation 8) does not fit the data satisfactorily. Adding a term to equation 8 which takes the power law nature [89] of the blinking of the QDs into account is seen to greatly improve the fit to the data. The auto-correlation function for translational diffusion and blinking two-photon excitation takes the form

$$
\begin{aligned}
& G(\tau)= \\
& G_{0}(\tau)\left[1+\frac{8 \tau_{D}}{r_{0}^{2}}\right]^{-1}\left[1+\frac{8 \tau_{D}}{z_{0}^{2}}\right]^{-1 / 2}\left[1+\frac{B}{1-B} \tau^{-\alpha}\right]
\end{aligned}
$$

where $B$ is the blinking amplitude and $\alpha$ is the exponent. The measured diffusion coefficient, of the QDs, found from a global analysis of the data using the model including the power law term was $19.7 \pm 2 \mu \mathrm{m}^{2} / \mathrm{sec}$. Using Stokes Einstein's equation, as in [76] we find the hydrodynamic radius to be $10.5 \pm 1 \mathrm{~nm}$.

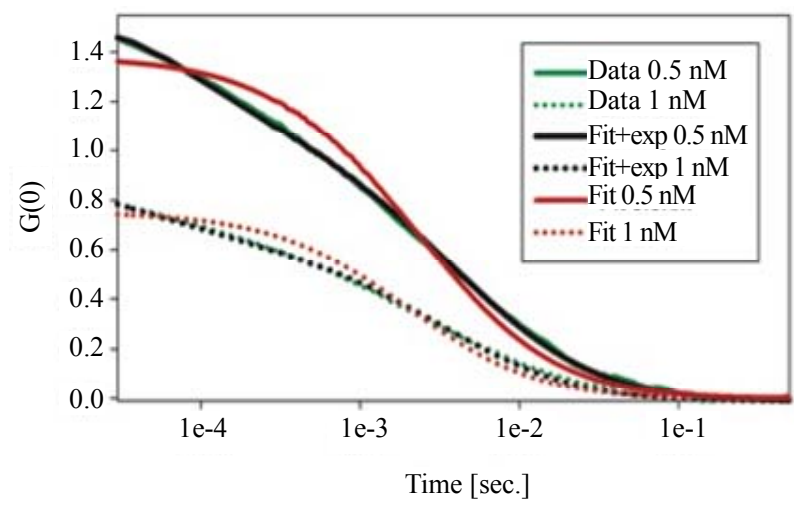

Figure 8. Auto correlation curves from FCS measurements carried out on $605 \mathrm{sAv}$ QDs from Invitrogen. The solid lines are for $0.5 \mathrm{nM}$ concentrations and the dotted lines are for 1 $n M$ concentrations of QDs. The green lines represent the data, the red lines are the best fit of a model for a single freely diffusing species. The black lines are the best fit of a model for a single freely diffusing molecule together with a power law term to fit the blinking. It is clear from the figure that a simple diffusion model does not completely describe the data. Adding a term which takes the blinking of the QDs into account, greatly improves the fit.

\section{Optical Trapping of Quantum Dots}

Optical tweezers are formed by tightly focusing a laser beam. Most often, optical tweezers are implemented in a microscope, equipped also with a quadrant photo-diode or a CCD for position detection with nanometer and micro-second resolution. Using an optical trap, particles in the nanometer to micrometer range can be manipulated in $3 \mathrm{D}$ and corresponding values of forces (typically in the pico-Newton range) and distances can be measured. An inducible dipole in an optical field experiences a force in the direction of the field gradient. This 3D restoring force is harmonic in all three dimension and directed towards the focus of the laser beam: $F=\kappa_{x}$, where $\kappa_{x}$ is denoted the trap stiffness in the $\mathrm{x}$-direction and $\mathrm{x}$ is the position of the particle with respect to the equilibrium position within the trap, the center of the potential sketched in Figure 9(a). From tracking the Brownian motion and knowledge of the size of the trapped object and the viscosity and temperature of the surrounding fluid, one can deduce the force, $\mathrm{F}$, acting on the trapped particle.

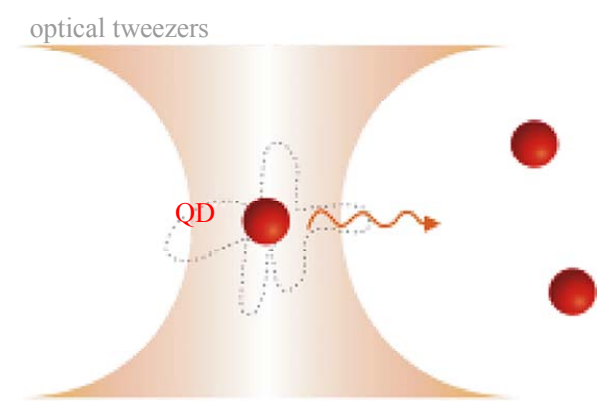

(a)

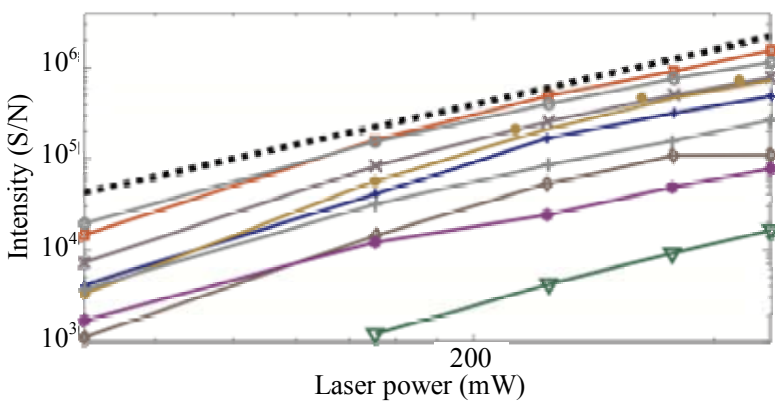

(b)

Figure 9. (a) Schematic of an optically trapped QD. The particle performs thermal motions in the harmonic potential while the QD is simultaneously two-photon excited and emits polarized red light. b) Emitted intensity (signal/noise) versus laser power for QD aggregates of varying initial size. The dotted line has a slope of 2 . The different aggregates have different initial intensities; probably because of their different sizes. Regardless of initial aggregate size, all traces scales with a slope of 2 ; the characteristic of two-photon absorption. Reprinted with permission from. Reprinted with permission from [90]. Copyright (2012) American Chemical Society. 
QDs, with inducible dipole moments, can be optically trapped by an infrared CW laser beam [91]. They serve as excellent combined force handles and visualization markers for investigations of individual cells, proteins, and biological filaments. In general, a fluorescent molecule can be two-photon excited if it simultaneously absorbs two photons, and if the energy difference between the ground and excited state of the molecule corresponds to the added value of the energies of the two photons. QDs can be excited by a two-photon absorption process [92,93], with a two-photon absorption cross section which is large in comparison to other fluorophores used in multiphoton microscopy [94-97]. Jauffred and Oddershede showed that QDs are readily excited through twophoton excitation by a relatively weak CW infrared laser beam, which can also be used to trap the QDs [98]. For a two-photon absorption the emitted intensity scales as $\propto$ $\mathrm{P}^{2}$, where $\mathrm{P}$ is the laser power, as shown in Figure 9(b).

One hallmark of optical trapping is that there exists a linear relationship between $\kappa$ and P. As shown in Figure 10(a), this is also true for optical trapping of two-photon excited QDs, thus supporting that the nonlinear absorption is only a weak perturbation that does not alter trapping properties considerably. It is important to correctly choose the QD such that it matches the given experimental goals and conditions, e.g., available excitation

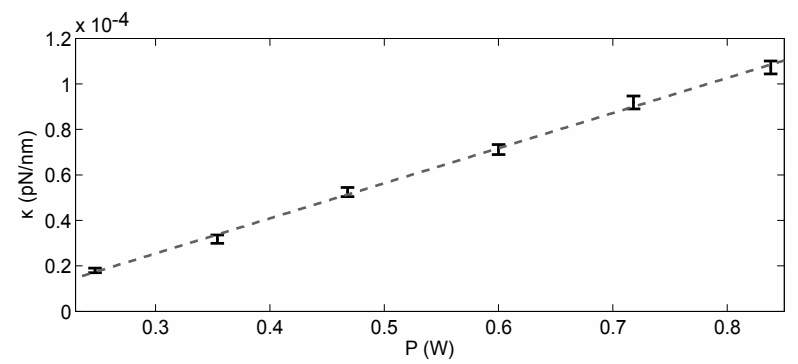

(a)

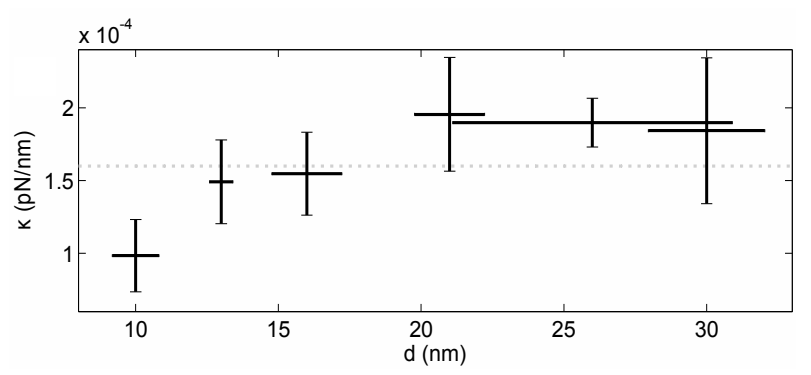

(b)

Figure 10. (a) Trapping strength versus laser power for individual QDs. The error bars denote one SEM. The dotted line is a linear fit to the data and it has a slope of $1.5 \times$ $20^{-3} \mathrm{pN} / \mathrm{nm} / \mathrm{W}$. Reprinted with permission from [90]. Copyright (2012) American Chemical Society. (b) Trapping strength dependence on diameters, $d$. All error bars denote one SEM and the dotted line is the mean value of $\kappa: \kappa=1.6$ $\times 10^{-4} \mathrm{pN} / \mathrm{nm}$. Reprinted with permission from [99]. lasers and filters. The trapping strength for QDs with different emission wavelengths $\lambda$ (from $525 \mathrm{~nm}$ to 800 $\mathrm{nm}$ ) and different physical sizes have similar trapping capabilities, as seen in Figure 10(b). The typical trapping strength measured for QDs is comparable to that obtained from optical trapping of silver and gold nanoparticles of similar sizes [100-104]. See Reference [105] for a review on optical tweezing and nanoparticles. In summary, the QD can serve both as a handle for manipulation and controlled force transduction and at the same time for visualization through two-photon absorption of the trapping laser light.

Optical trapping relies on light scattering, a linear property. Within the recent years efforts were done also to take advantage of the angular momentum of the laser beam $[106,107]$.

Angular momentum is transferred to the trapped particle by changing the direction of the laser polarization vector. Most often optical tweezers are based on linearly polarized light and an elongated trapped object, smaller than the diffraction limit, will align with the laser's polarization vector, as shown in Figure 11(a). Objects larger than the diffraction limit, as for instance bacteria, will align along the propagation direction of the trapping laser. By changing the direction of the laser's polarization vector, e.g., by using circularly polarized light, a trapped elongated nano-particle can be forced to rotate in a controlled fashion.

This was successfully done by Head et al. [108], who used a focused laser bam to trap and two-photon excite elongated quantum rods (with an aspect ratio of 10). They used circularly polarized light and hence, the trapped quantum rod would rotate in an oscillatory manner. The quantum rod emitted light polarized along its long axis and by analyzing emitted light using a polarization filter (as sketched in Figure 11(b)), they were able to

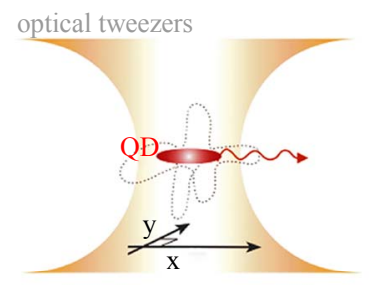

(a)

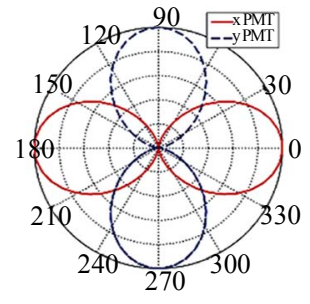

(b)
Figure 11. (a) Schematic of an optically trapped quantum rod that aligns along the laser polarization but still perform significant 3D Brownian; (b) Schematic of the polarized emission from an optically trapped semiconductor quantum rod. The light emitted from the trapped quantum rod is polarized along its long axis. The polar intensity plot shows the light ideally measured by two orthogonally polarized photomultipliers ( $x$-PMT and $y$-PMT) as a function of the polarization angle measured from the $x$-axis, this is indeed consistent with experimental measurements [108]. 
detect that the trapped quantum rod was indeed rotating with the same frequency as the applied polarization vector was changing with. The trapped particle could follow field rotations of, at least, $320 \mathrm{~Hz}$ [108]. These results pave the way for a system where the trapping and visualization is further combined with the ability to measure and transduce torque.

Altogether, with a single infrared tightly focused laser beam one can optically trap, rotate, and perform twophoton excitation of individual colloidal QDs. Hence, individual quantum dots serve both as force and torque tranducers as well as visualization markers.

\section{Conclusion}

In this review we demonstrated the versatility of using individual quantum dots as markers for individual molecules and as force probes. QDs can be advantageously applied for single particle tracking, where their low bleaching rates make them favorable in comparison to conventional fluorophores. Also, they give clear results in FCS experiments, and can be individually optically trapped, thus serving as force and torque transducing handles. The great spectral properties of QDs include high photostability, narrow emission spectra, blinking and low bleaching rate. The photo-physical properties of QDs make them ideal for multicolour experimental setups, since several differently coloured QDs can be distinguished at the same time, while being illuminated by a single light source. When performing single particle tracking experiments, the blinking of QDs is a disadvantage as certain visited positions remain undetected. However, blinking is also an advantage in localization microscopy, in particular in the novel super-resolution techniques, as a subset of the QDs will emit in each image frame. For super-resolution localization microscopy QDs have a high potential in experiments involving position localization microscopy of lipids and proteins in the plasma membrane in live mammalian cells [7,109]. In live-cell experiments as well as in vivo experiments in animals, QDs have a strong potential as fluorescent probe as they can be easily inserted into the cytoplasm and then individually detected, conjugated and optically manipulated [37,110-118]. In FCS experiments, the great advantages of QDs are their well defined size and high fluorescence emission, which make QDs a trust-worthy reference or a good label of membrane proteins or lipids. The possibility to excite QDs with two-photon excitation also makes QDs applicable in skin permeability or drug uptake receptor studies in which deep tissue microscopy is necessary. Finally, as QDs can be individually manipulated and visualized using a single laser beam, they serve as the optimal handle and marker for the expanding efforts in uncovering the action of individual molecules and for designed nano-scopic molecular electronics.

\section{Acknowledgements}

We thank The Danish Council for Independent Research for grant to EAC and funding from The Danish Research Infrastructure to the Danish Molecular Biomedical Imaging Center and Dr. Mathias Clausen for reading the manuscript before submission.

\section{REFERENCES}

[1] M. Bruchez, et al., Science, Vol. 281, 1998, pp. 20132016. http://dx.doi.org/10.1126/science.281.5385.2013

[2] X. Gao, et al., Current Opinion in Biotechnology, Vol. 16, 2005, pp. 63-72.

http://dx.doi.org/10.1016/j.copbio.2004.11.003

[3] B. O. Dabbousi, et al., The Journal of Physical Chemistry $B$, Vol. 101, 1997, pp. 9463-9475. http://dx.doi.org/10.1021/jp971091y

[4] M. A. Hines and P. Guyot-Sionnest, The Journal of Physical Chemistry, Vol. 100, 1996, pp. 468-471. http://dx.doi.org/10.1021/jp9530562

[5] T. Kippeny, L. A. Swafford and S. J. Rosenthal, Journal of Chemical Education, Vol. 79, 2002, pp. 1094-1100. http://dx.doi.org/10.1021/ed079p1094

[6] Z. Deng, et al., Journal of the American Chemical Society, Vol. 134, 2012, pp. 17424-17427. http://dx.doi.org/10.1021/ja3081023

[7] B. C. Lagerholm, et al., Biophysical Journal, Vol. 91, 2006, pp. 3050-3060. http://dx.doi.org/10.1529/biophysj.105.079178

[8] S. F. Lee and M. A. Osborne, ChemPhyschem, Vol. 10, 2009, pp. 2174-2191. http://dx.doi.org/10.1002/cphc.200900200

[9] S. Hohng and T. Ha, Journal of the American Chemical Society, Vol. 126, 2004, pp. 1324-1325. http://dx.doi.org/10.1021/ja039686w

[10] W. E. Moerner and M. Orrit, Science, Vol. 283, 1999, pp. 1670-1676. http://dx.doi.org/10.1126/science.283.5408.1670

[11] M. Nirmal, et al., Nature, Vol. 383, 1996, pp. 802-804. http://dx.doi.org/10.1038/383802a0

[12] N. Durisic, et al., ACS Nano, Vol. 3, 2009, pp. 1167-1175. http://dx.doi.org/10.1021/nn800684z

[13] N. Durisic, et al., Biophysical Journal, Vol. 93, 2007, pp. 1338-1346. http://dx.doi.org/10.1529/biophysj.107.106864

[14] X. Wang, et al., Nature, Vol. 459, 2009, pp. 686-689. http://dx.doi.org/10.1038/nature08072

[15] A. Biebricher, M. Sauer and P. Tinnefeld, The Journal of Physical Chemistry B, Vol. 110, 2006, pp. 5174-5178. http://dx.doi.org/10.1021/jp060660b

[16] V. Fomenko and D. J. Nesbitt, Nano Letters, Vol. 8, 2008, pp. 287-293. http://dx.doi.org/10.1021/n10726609

[17] Y. Chen, et al., Journal of the American Chemical Society, Vol. 130, 2008, pp. 5026-5027. http://dx.doi.org/10.1021/ja711379k 
[18] B. Mahler, et al., Nature Materials, Vol. 7, 2008, pp. 659-664. http://dx.doi.org/10.1038/nmat2222

[19] M. Nirmal, et al., Physical Review Letters, Vol. 75, 1995, pp. 3728-3731. http://dx.doi.org/10.1103/PhysRevLett.75.3728

[20] K. T. Shimizu, et al., Physical Review B, Vol. 6320, 2001, Article ID: 205316.

[21] P. A. Frantsuzov and R. A. Marcus, Physical Review B, Vol. 72, 2005, Article ID: 155321. http://dx.doi.org/10.1103/PhysRevB.72.155321

[22] X. Brokmann, et al., Physical Review Letters, Vol. 90, 2003, Article ID: 120601. http://dx.doi.org/10.1103/PhysRevLett.90.120601

[23] M. Kuno, et al., Journal of Chemical Physics, Vol. 115, 2001, pp. 1028-1040. http://dx.doi.org/10.1063/1.1377883

[24] M. Kuno, et al., The Journal of Chemical Physics, Vol. 112, 2000, pp. 3117-3120. http://dx.doi.org/10.1063/1.480896

[25] L. Wang, The Journal of Physical Chemistry B, Vol. 105, 2001, pp. 2360-2364. http://dx.doi.org/10.1021/jp0032053

[26] A. L. Efros and M. Rosen, Physical Review Letters, Vol. 78, 1997, pp. 1110-1113. http://dx.doi.org/10.1103/PhysRevLett.78.1110

[27] S. F. Lee and M. A. Osborne, Journal of the American Chemical Society, Vol. 129, 2007, pp. 8936-8937. http://dx.doi.org/10.1021/ja071876+

[28] W. G. J. H. M. van Sark, et al., The Journal of Physical Chemistry B, Vol. 105, 2001, pp. 8281-8284. http://dx.doi.org/10.1021/jp012018h

[29] H. Chen, H. Gai and E. S. Yeung, Chemical Communications, No. 13, 2009, pp. 1676-1678. http://dx.doi.org/10.1039/b819356h

[30] J. E. B. Katari, V. L. Colvin and A. P. Alivisatos, The Journal of Physical Chemistry, Vol. 98, 1994, pp. 41094117. http://dx.doi.org/10.1021/j100066a034

[31] P. Hoyer, et al., Nano Letters, Vol. 11, 2011, pp. 245-250. http://dx.doi.org/10.1021/nl103639f

[32] E. A. Christensen, P. Kulatunga and B. C. Lagerholm, PLoS One, Vol. 7, 2012, Article ID: e44355. http://dx.doi.org/10.1371/journal.pone.0044355

[33] Y. Xing, Z. Xia and J. Rao, IEEE Transactions on NanoBioscience, Vol. 8, 2009, pp. 4-12. http://dx.doi.org/10.1109/TNB.2009.2017321

[34] X. Michalet, et al., Science, Vol. 307, 2005, pp. 538-544. http://dx.doi.org/10.1126/science.1104274

[35] T. Pons and H. Mattoussi, Annals of Biomedical Engineering, Vol. 37, 2009, pp. 1934-1959. http://dx.doi.org/10.1007/s10439-009-9715-0

[36] M. Bruchez Jr., M. Moronne, P. Gin, S. Weiss and A. P. Alivisatos, Science, Vol. 281, 1998, pp. 2013-2016. http://dx.doi.org/10.1126/science.281.5385.2013

[37] B. Dubertret, P. Skourides, D. J. Norris, V. Noireaux, A. H. Brivanlou and A. Libchaber, Science, Vol. 298, 2002, pp. 1759-1762. http://dx.doi.org/10.1126/science.1077194

[38] X. Y. Wu, H. J. Liu, J. Q. Liu, K. N. Haley, J. A. Treadway, J P. Larson, N. F. Ge, F. Peale and M. P. Bruchez, Nature Biotechnology, Vol. 21, 2002, pp. 41-46. http://dx.doi.org/10.1038/nbt764

[39] T. Pellegrino, L. Manna, S. Kudera, T. Liedl, D. Koktysh, A. L. Rogach, S. Keller, J. Rädler, G. Natile and W. J. Parak, Nano Letters, Vol. 4, 2004, pp. 703-707. http://dx.doi.org/10.1021/n1035172j

[40] S. J. Rosenthal, J. C. Chang, O. Kovtun, J. R. McBride and I. D. Tomlinson, Chemistry \& Biology, Vol. 18, 2011, pp. $10-24$ http://dx.doi.org/10.1016/i.chembiol.2010.11.013

[41] G. T. Hermanson, "Bioconjugate Techniques," Academic Press, San Diego, 1996.

[42] I. L. Medintz, H. T. Uyeda, E. R. Goldman and H. Mattoussi, Nature Materials, Vol. 4, 2005, pp. 435-446. http://dx.doi.org/10.1038/nmat1390

[43] S. K. Chakraborty, J. A. J. Fitzpatrick, J. A. Phillippi, S. Andreko, A. S. Waggoner, M. P. Bruchez and B. Ballou, Nano Letters, Vol. 7, 2007, pp. 2618-2626. http://dx.doi.org/10.1021/nl0709930

[44] S. Pathak, M. C. Davidson and G. A. Silva, Nano Letters, Vol. 7, 2007, pp. 1839-1345. http://dx.doi.org/10.1021/n1062706i

[45] T. Jamieson, R. Bakhshia, D. Petrovaa, R. Pococka, M. Iman and A. M. Seifalian, Biomaterials, Vol. 28, 2007, pp. 4717-4732. http://dx.doi.org/10.1016/j.biomaterials.2007.07.014

[46] E. L. Bentzen, I. D. Tomlinson, J. Mason, P. Gresch, M. R. Warnement, D. Wright, E. Sanders-Bush, R. Blakely and S. J. Rosenthal, Bioconjugate Chemistry, Vol. 16, 2005, pp. 1488-1494. http://dx.doi.org/10.1021/bc0502006

[47] W. R. Algar, A. J. Tavares and U. J. Krull, Analytica Chimica Acta, Vol. 673, 2010, pp. 1-25. http://dx.doi.org/10.1016/j.aca.2010.05.026

[48] D. Alcor, G. Gouzer and A. Triller, European Journal of Neuroscience, Vol. 30, 2009, pp. 987-997. http://dx.doi.org/10.1111/j.1460-9568.2009.06927.x

[49] M. J. Saxton and K. Jacobson, Annual Review of Biophysics and Biomolecular Structure, Vol. 26, 1997, pp. 373-399. http://dx.doi.org/10.1146/annurev.biophys.26.1.373

[50] C. Joo, H. Balci, Y. Ishitsuka, C. Buranachai and T. Ha, Annual Review of Biochemistry, Vol. 77, 2008, pp. 51-76. http://dx.doi.org/10.1146/annurev.biochem.77.070606.10 $\underline{1543}$

[51] M. Brameshuber and G. J. Schutz, Nature Methods, Vol. 5, 2008, pp. 133-134. http://dx.doi.org/10.1038/nmeth0208-133

[52] M. P. Clausen and B. C. Lagerholm, Current Protein \& Peptide Science, Vol. 12, 2011, pp. 699-713.

[53] F. Pinaud, S. Clarke, A. Sittner and M. Dahan, Nature Methods, Vol. 7, 2010, pp. 275-285. http://dx.doi.org/10.1038/nmeth.1444

[54] S. Wieser and G. J. Schutz, Methods, Vol. 46, 2008, pp. 
131-140. http://dx.doi.org/10.1016/j.ymeth.2008.06.010

[55] H. Bannai, S. Lévi, C. Schweizer, M. Dahan and A. Triller, Nature Protocols, Vol. 1, 2006, pp. 2628-2634. http://dx.doi.org/10.1038/nprot.2006.429

[56] A. Serge, N. Bertaux, H. Rigneault and D. Marguet, $N a-$ ture Methods, Vol. 5, 2008, pp. 687-694. http://dx.doi.org/10.1038/nmeth.1233

[57] K. Jaqaman, D. Loerke, M. Mettlen, H. Kuwata, S. Grinstein, S. L. Schmid and G. Danuser, Nature Methods, Vol. 5, 2008, pp. 695-702. http://dx.doi.org/10.1038/nmeth.1237

[58] I. F. Sbalzarini and P. Koumoutsakos, Journal of Structural Biology, Vol. 151, 2005, pp. 182-195. http://dx.doi.org/10.1016/j.jsb.2005.06.002

[59] J. Adler, A. I. Shevchuk, P. Novak, Y. E. Korchev and I. Parmryd, Nature Methods, Vol. 7, 2010, pp. 170-171. http://dx.doi.org/10.1038/nmeth0310-170

[60] A. Kusumi, Y. M. Shirai, I. Koyama-Honda, K. G. N. Suzuki and T. K. Fujiwara, FEBS Letters, Vol. 584, 2010, pp. 1814-1823. http://dx.doi.org/10.1016/i.febslet.2010.02.047

[61] D. Lingwood and K. Simons, Science, Vol. 327, 2010, pp. 46-50. http://dx.doi.org/10.1126/science.1174621

[62] P. S. Niemela, M. S. Miettinen, L. Monticelli, H. Hammaren, P. Bjelkmar, T. Murtola, E. Lindahl and I. Vattulainen, Journal of the American Chemical Society, Vol. 132, 2010, pp. 7574-7575. http://dx.doi.org/10.1021/ja101481b

[63] M. Dahan, S. Lévi, C. Luccardini, P. Rostaing, B. Riveau and A. Triller, Science, Vol. 302, 2003, pp. 442-445. http://dx.doi.org/10.1126/science.1088525

[64] N. L. Andrews, K. A. Lidke, J. R. Pfeiffer, A. R. Burns, B. S. Wilson, J. M. Oliver and D. S. Lidke, Nature Cell Biology, Vol. 10, 2008, pp. 955-963. http://dx.doi.org/10.1038/ncb1755

[65] R. Frischknecht, M. Heine, D. Perrais, C. I. Seidenbecher, D. Choquet and E. D. Gundelfinger, Nature Neuroscience, Vol. 12, 2009, pp. 897-904. http://dx.doi.org/10.1038/nn.2338

[66] F. Pinaud, X. Michalet, G. Iyer, E. Margeat, H.-P. Moore and S. Weiss, Traffic, Vol. 10, 2009, pp. 691-712. http://dx.doi.org/10.1111/j.1600-0854.2009.00902.x

[67] I. R. Bates, B. Hébert, Y. S. Luo, J. Liao, A. I. Bachir, D. L. Kolin, P. W. Wiseman and J. W. Hanrahan, Biophysical Journal, Vol. 91, 2006, pp. 1046-1058. http://dx.doi.org/10.1529/biophysj.106.084830

[68] A. Triller and D. Choquet, Neuron, Vol. 59, 2008, pp. 359-374. http://dx.doi.org/10.1016/j.neuron.2008.06.022

[69] C. Bouzigues, M. Morel, A. Triller and M. Dahan, Proceedings of the National Academy of Sciences of the United States of America, Vol. 104, 2007, pp. 11251-11256. http://dx.doi.org/10.1073/pnas.0702536104

[70] M. Heine, L. Groc, R. Frischknecht, J.-C. Béïque, B. Lounis, G. Rumbaugh, R. L. Huganir, L. Cognet and D. Choquet, Science, Vol. 320, 2008, pp. 201-205. http://dx.doi.org/10.1126/science.1152089

[71] D. S. Lidke, K. A. Lidke, B. Rieger, T. M. Jovin and D. J.
Arndt-Jovin, Journal of Cell Biology, Vol. 170, 2005, pp. 619-626. http://dx.doi.org/10.1083/jcb.200503140

[72] D. S. Lidke, P. Nagy, R. Heintzmann, D. J. Arndt-Jovin, J. N. Post, H. E. Grecco, E. A. Jares-Erijman and T. M. Jovin, Nature Biotechnology, Vol. 22, 2004, pp. 198-203. http://dx.doi.org/10.1038/nbt929

[73] S. T. Low-Nam, K. A. Lidke, P. J. Cutler, R. C. Roovers, P. M. van Bergen en Henegouwen, B. S. Wilson and D. S. Lidke, Nature Structural and Molecular Biology, Vol. 18, 2011, pp. 1244-1249.

http://dx.doi.org/10.1038/nsmb.2135

[74] C. J. You, S. Wilmes, O. Beutel, S. Löchte, Y. Podoplelowa, F. Roder, C. Richter, T. Seine, D. Schaible, G. Uzé, S. Clarke, F. Pinaud, M. Dahan and J. Piehler, Angewandte Chemie International Edition, Vol. 49, 2010, pp. 4108-4112. http://dx.doi.org/10.1002/anie.200907032

[75] N. L. Andrews, J. R. Pfeiffer, A. M. Martinez, D. M. Haaland, R. W. Davis, T. Kawakami, J. M. Oliver, B. S. Wilson and D. S. Lidke, Immunity, Vol. 31, 2009, pp. 469-479. http://dx.doi.org/10.1016/j.immuni.2009.06.026

[76] E. C. Arnspang, J. R. Brewer and B. C. Lagerholm, PLoS ONE, Vol. 7, 2012, Article ID: e48521. http://dx.doi.org/10.1371/journal.pone.0048521

[77] M. P. Clausen and B. C. Lagerholm, Nano Letters, Vol. 13, 2013, pp. 2332-2337. http://dx.doi.org/10.1021/nl303151f

[78] K. Rohr and S. Vogel, Chembiochem: A European Journal of Chemical Biology, Vol. 7, 2006, pp. 463-470. http://dx.doi.org/10.1002/cbic.200500392

[79] A. C. Simonsen and L. A. Bagatolli, Langmuir: The ACS Journal of Surfaces and Colloids, Vol. 20, 2004, pp. 9720-9728. http://dx.doi.org/10.1021/la048683+

[80] K. Braeckmans, K. Remaut, R. E. Vandenbroucke, B. Lucas, S. C. De Smedt and J. Demeester, Biophysical Journal, Vol. 92, 2007, pp. 2172-2183. http://dx.doi.org/10.1529/biophysj.106.099838

[81] D. Magde, W. W. Webb and E. Elson, Physical Review Letters, Vol. 29, 1972, pp. 705-708. http://dx.doi.org/10.1103/PhysRevLett.29.705

[82] E. L. Elson, Journal of Biomedical Optics, Vol. 9, 2004, pp. 857-864. http://dx.doi.org/10.1117/1.1779234

[83] K. M. Berland, P. T. C. So and E. Gratton, Biophysical Journal, Vol. 68, 1995, pp. 694-701. http://dx.doi.org/10.1016/S0006-3495(95)80230-4

[84] E. Haustein and P. Schwille, Annual Review of Biophysics and Biomolecular Structure, Vol. 36, 2007, pp. 151-169. http://dx.doi.org/10.1146/annurev.biophys.36.040306.132 $\underline{612}$

[85] P. Schwille, U. Haupts, S. Maiti and W. W. Webb, Biophysical Journal, Vol. 77, 1999, pp. 2251-2265. http://dx.doi.org/10.1016/S0006-3495(99)77065-7

[86] E. Haustein and P. Schwille, Current Opinion in Structural Biology, Vol. 14, 2004, pp. 531-540. http://dx.doi.org/10.1016/j.sbi.2004.09.004

[87] A. Einstein, Annalen der Physik, Vol. 322, 1905, pp. 549560. http://dx.doi.org/10.1002/andp.19053220806

[88] J. Brewer, J. B. de la Serna, K. Wagner and L. A. Baga- 
tolli, Biochimica et Biophysica Acta, Vol. 1798, 2010, pp. 1301-1308.

http://dx.doi.org/10.1016/j.bbamem.2010.02.024

[89] A. I. Bachir, N. Durisic, B. Hebert, P. Grütter and P. W. Wiseman, Journal of Applied Physics, Vol. 99, 2006, Article ID: 064503. http://dx.doi.org/10.1063/1.2175470

[90] L. Jauffred and L. B. Oddershede, Nano Letters, Vol. 10, 2010, pp. 1927-1930. http://dx.doi.org/10.1021/nl100924z

[91] L. Jauffred, A. C. Richardson and L. B. Oddershede, Nano letters, Vol. 8, 2008, pp. 3376-3380. http://dx.doi.org/10.1021/nl801962f

[92] M. Schmidt, S. A. Blanton, M. A. Hines and P. GuyotSionnest,. Physical Review B, Vol. 53, 1996, pp. 1262912632. http://dx.doi.org/10.1103/PhysRevB.53.12629

[93] S. A. Blanton, A. Dehestani, P. C. Lin and P. Guyot-Sionnest, Chemical Physics Letters, Vol. 229, 1994, pp. 317322. http://dx.doi.org/10.1016/0009-2614(94)01057-9

[94] T. Wang, J. Y. Chen, S. Zhen, P. N. Wang, C. C. Wang, W. L. Yang and Q. Peng, Journal of Fluorescence, Vol. 19, 2009, pp. 615-621. http://dx.doi.org/10.1007/s10895-008-0452-9

[95] L. Pan, A. Ishikawa and N. Tamai, Physical Review B, Vol. 75, 2007, p. 161305. http://dx.doi.org/10.1103/PhysRevB.75.161305

[96] S. C. Pu, M. J. Yang, C.-C. Hsu, C.-W. Lai, C.-C. Hsieh, S. H. Lin, Y. M. Cheng and P. T. Chou, Small, Vol. 2, 2006, pp. 1308-1313. http://dx.doi.org/10.1002/smll.200600157

[97] D. R. Larson, W. R. Zipfel, R. M. Williams, S. W. Clark, M. P. Bruchez, F. W. Wise and W. W. Webb, Science, Vol. 300, 2003, pp. 1434-1436. http://dx.doi.org/10.1126/science. 1083780

[98] L. Jauffred and L. B. Oddershede, Nano Letters, Vol. 10, 2010, pp. 1927-1930. http://dx.doi.org/10.1021/nl100924z

[99] L. Jauffred, M. Sletmoen, F. Czerwinski and L. Oddershede, Quantum dots as handles for optical manipulation. arXiv.org, 2010. physics.optics.

[100] F. Hajizadeh and S. N. S. Reihani, Optics Express, Vol. 18, 2010, pp. 551-559. http://dx.doi.org/10.1364/OE.18.000551

[101] C. Selhuber-Unkel, I. Zins, O. Schubert and C. Sönnichsen, Nano letters, Vol. 8, 2008, pp. 2998-3003. http://dx.doi.org/10.1021/n1802053h

[102] L. Bosanac, T. Aabo, P. M. Bendix and L. B. Oddershede, Nano letters, Vol. 8, 2008, pp. 1486-1491. http://dx.doi.org/10.1021/n1080490+

[103] P. M. Hansen, V. K. Bhatia, N. Harrit and L. Oddershede, Nano letters, Vol. 5, 2005, pp. 1937-1942. http://dx.doi.org/10.1021/n1051289r

[104] K. Svoboda and S. M. Block, Optics Letters, Vol. 19, 1994, pp. 930-932.

http://dx.doi.org/10.1364/OL.19.000930

[105] M. Dienerowitz, M. Mazilu and K. Dholakia, Journal of Nanophotonics, Vol. 2, 2008, Article ID: 021875. http://dx.doi.org/10.1117/1.2992045

[106] M. Padgett and R. Bowman, Nature Photonics, Vol. 5, 2011, pp. 343-348. http://dx.doi.org/10.1038/nphoton.2011.81

[107] K. Dholakia and T. Čižmár, Nature Photonics, Vol. 5, 2011, pp. 335-342. http://dx.doi.org/10.1038/nphoton.2011.80

[108] C. R. Head, E. Kammann, M. Zanella, L. Mannabc and P. G. Lagoudakis, Nanoscale, Vol. 4, 2012, pp. 3693-3697. http://dx.doi.org/10.1039/c2nr30515a

[109] K. Lidke, B. Rieger, T. Jovin and R. Heintzmann, Optics Express, Vol. 13, 2005, pp. 7052-7062. http://dx.doi.org/10.1364/OPEX.13.007052

[110] P. Pierobon, S. Achouri, S. Courty, A. R. Dunn, J. A. Spudich, M. Dahan and G. Cappello, Biophysical Journal, Vol. 96, 2009, pp. 4268-4275. http://dx.doi.org/10.1016/j.bpj.2009.02.045

[111] C. Kural, H. Kim, S. Syed, G. Goshima, V. I. Gelfand and P. R. Selvin, Science, Vol. 308, 2005, pp. 1469-1472. http://dx.doi.org/10.1126/science. 1108408

[112] L. B. Oddershede, Nature Chemical Biology, Vol. 8, 2012, pp. 879-886. http://dx.doi.org/10.1038/nchembio.1082

[113] A. Biebricher, W. Wende, C. Escudé, A. Pingoud and P. Desbiolles, Biophysical Journal, Vol. 96, 2009, pp. L50L52. http://dx.doi.org/10.1016/j.bpj.2009.01.035

[114] Y. Ebenstein, N. Gassman, S. Kim, J. Antelman, Y. Kim, S. Ho, R. Samuel, X. Michalet and S. Weiss, Nano Letters, Vol. 9, 2009, pp. 1598-1603. http://dx.doi.org/10.1021/n1803820b

[115] I. J. Finkelstein, M. L. Visnapuu and E. C. Greene, $\mathrm{Na}$ ture, Vol. 468, 2010, pp. 983-987. http://dx.doi.org/10.1038/nature09561

[116] A. Seitz and T. Surrey, The EMBO Journal, Vol. 25, 2006, pp. 267-277. http://dx.doi.org/10.1038/sj.emboj.7600937

[117] D. M. Warshaw, G. G. Kennedy, S. S. Work, E. B. Krementsova, S. Beck and K. M. Trybus, Biophysical Journal, Vol. 88, 2005, pp. L30-L32. http://dx.doi.org/10.1529/biophysj.105.061903

[118] M. Eriksen, P. Horvath, M. A. Sørensen, S. Semsey, L. B. Oddershede and L. Jauffred, Journal of Nanomaterials, Vol. 2013, 2013, Article ID: 468105. http://dx.doi.org/10.1155/2013/468105 